\title{
Development of slow and fast wave coupling and heating from the C-Stellarator to NSTX
}

\author{
Joel Hosea ${ }^{1, *}$ and the PPPL ICRF Teams \\ ${ }^{1}$ Princeton Plasma Physics Laboratory, Princeton University, Princeton, NJ 08543, USA
}

\begin{abstract}
A historical perspective on key discoveries which contributed to understanding the properties of coupling both slow and fast waves and the effects on plasma heating and current drive will be presented. Important steps made include the demonstration that the Alfven resonance was in fact a mode conversion on the $\mathrm{C}$-stellarator, that toroidal $\mathrm{m}=-1$ eigenmodes were excited in toroidal geometry and impurity influx caused the $Z$ mode on the ST tokamak, that the $\mathrm{H}$ minority regime provided strong heating and that ${ }^{3} \mathrm{He}$ minority could be used as well on $\mathrm{PLT}$, that the $2^{\text {nd }}$ harmonic majority tritium regime was viable on TFTR, and that high harmonic fast wave heating was efficient when the SOL losses were avoided on NSTX.
\end{abstract}

\section{Introduction}

Understanding of ICRF coupling and heating physics has evolved through the combination of experiments, modeling, and technology over several decades. At PPPL this has involved experiments and modeling for a line of experimental devices including $-\mathrm{C}$ stellarator, ST tokamak, PLT tokamak, TFTR tokamak, and NSTX/NSTX-U spherical tokamak - and employing critical hardware and diagnostics for achieving high power operation and observing the RF effects on the core and scrape off layer (SOL) plasmas. It is informative to track the historical evolution of advances in understanding on these devices and a selection of these advances is given here.

\section{C stellarator: Alfven "resonance" shown to be an Alfven mode conversion}

The $\mathrm{C}$ stellarator was configured in a race track geometry with the ICRF four strap antenna located in one straight section and a toroidal limiter located in the other straight section [1]. Thus the waves were launched in a purely cylindrical region, propagated away from the antenna, and damped in the magnetic beaches [2].

Early ICRF experiments on the $\mathrm{C}$ stellarator showed that the antenna loading was up to $\sim 6$ times larger than predicted with an approximate model for the loading [2], and this mysterious loading was designated as mode $\mathrm{X}$ [3]. The technology addition of a faraday shield between the antenna straps and the plasma removed this mode $\mathrm{X}$ loading [3] and opened the way to comparing experimental loading predictions to quantitative predictions from a cylindrical model utilizing the full

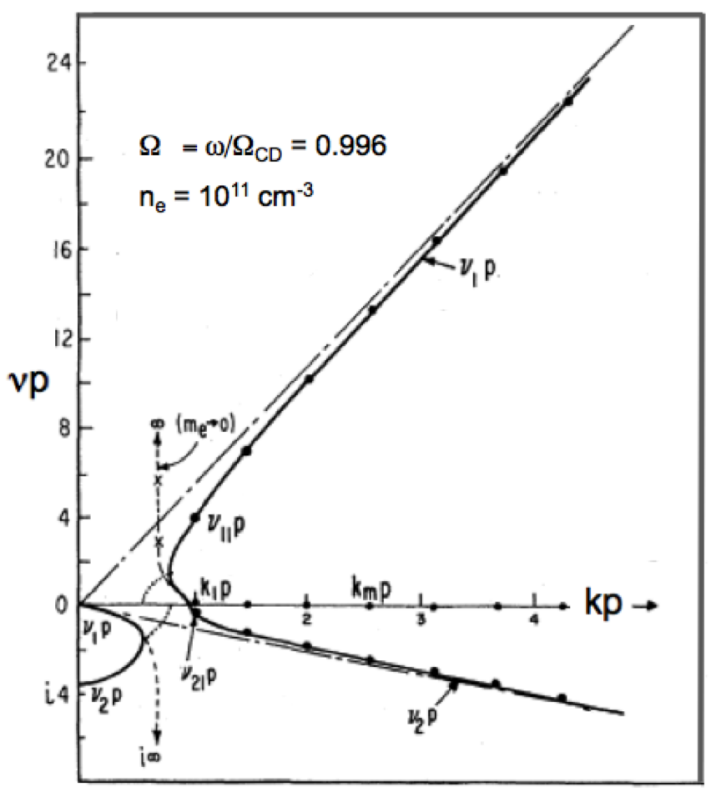

Figure 1. Alfven mode conversion of perpendicular wavenumber $v p$ versus parallel wavenumber $\mathrm{kp}$ for Model-C parameters for $\Omega<1$. (Plasma radius $\mathrm{p}=6.1 \mathrm{~cm}$.) (Fig. 1 of Ref. 4.)

cold plasma relation. The first step for developing this model was recognizing that the "Alfven resonance" was in fact an "Alfven mode conversion" even in the absence of finite temperature as shown in Fig. 1 [4,5]. When it was assumed earlier that the electron mass could be set to zero and the essential wave physics would be preserved, an artificial resonance resulted. But keeping the electron mass in the plasma dispersion indeed gives the mode conversion at $\mathrm{n}^{2}{ }_{\|}=\mathrm{S}$ (T. Stix notation [2]) for this regime with $\omega \prec \Omega_{\text {ci }}$. The orthogonal eigenmodes

*Corresponding author: jhosea@pppl.gov 


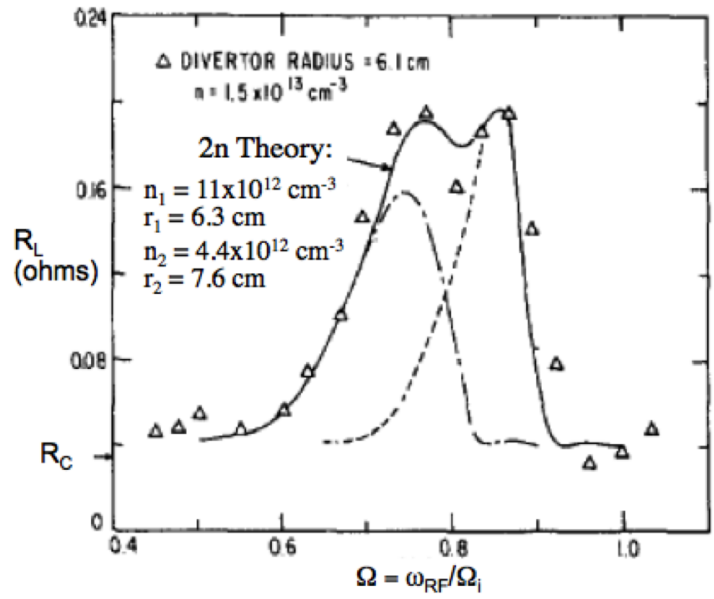

Figure 2. Experimental loading of Stix coil versus $\Omega$, and its prediction by the two-density cold plasma theory. (Fig. 3 of Ref. 6 with AIP Publishing permission)
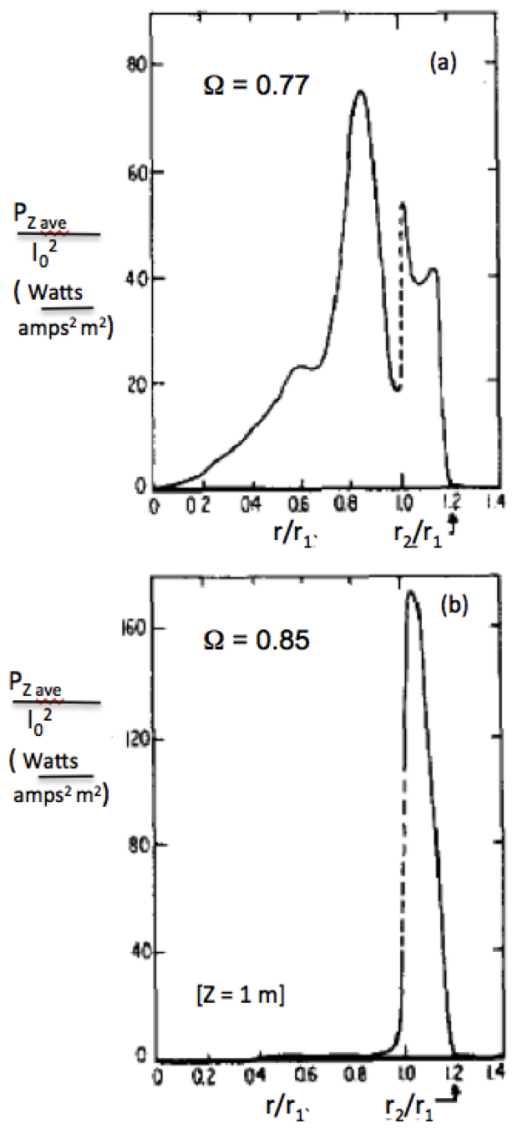

Figure 3. The average Poynting flux in the axial direction $1 \mathrm{~m}$ along the axis from the center of the Stix coil for the two peaks in Fig, 2. (Fig. 4 of Ref.6 with AIP Publishing permission)

which fit in the cylindrical model straight section have two radial mode numbers for each parallel wavenumber one on the "electrostatic" arm and one on the "electromagnetic" arm - on the right branch in Fig. 1. It should be noted that all of the modes fall on the slow wave branch since fast wave modes were not excited under the $\omega / \Omega_{\mathrm{ci}} \prec 1$ conditions on the $\mathrm{C}$ stellarator. Subsequently, the loading for each of the modes could be calculated with the residue method described in Ref. 5 and summed over the antenna spectrum, which peaked at $\mathrm{k}_{\|} \mathrm{p} \sim 1$, to compare to the measured loading.

To obtain sharper loading profiles in $\Omega=\omega / \Omega_{\mathrm{ci}}$ a second technology step was taken to make the magnetic field very close to constant over the antenna region [4]. The theoretical $\mathrm{R}_{\mathrm{L}}$ loading profiles versus $\Omega$ followed the experimental profiles quantitatively over the entire range of densities studied - from $1.5 \times 10^{18} \mathrm{~m}^{-3}$ to $2 \mathrm{x}$ $10^{16} \mathrm{~m}^{-3}$ [4]. Importantly, the maximum of $\mathrm{R}_{\mathrm{L}}$ falls off as $\Omega=1$ is approached in contrast to the approximate prediction with $\mathrm{m}_{\mathrm{e}}=0$. This comparison clearly verifies the cold plasma theory with the Alfven mode conversion. Subsequently, this modeling was extended to a two step density profile that accounted for the density drop-off in the $\mathrm{C}$ stellarator toroidal divertor and used to explain the two peak loading profile versus $\Omega$ observed at higher density (Fig. 2) [6]. This comparison further revealed that the peak closest to $\Omega=1$ was due to power coupled to the SOL as shown in Fig.3, and such coupling can be a major effect in the fast-wave regime on NSTX as will be discussed later.

\section{ST tokamak: Transition to toroidal geometry and fast wave excitation}

The C stellarator was converted to the ST tokamak in 1970 with the toroidal configuration being provided by removing its straight sections. This forced us to rethink the wave launching and wave damping which overlap in the same cross-section in a torus. We adopted the strategy of launching slow and fast waves from the inside of the plasma torus with a half-turn current strap. We charged ahead with a cylindrical plasma model to provide an approximate analysis of both the slow wave and fast wave excitation [7]. Eigenmodes were predicted for both slow and fast waves with poloidal $\mathrm{m}$ values of $0,+1$, and -1 . One question at the time was do distinct toroidal eigenmodes exist in toroidal geometry or are the modes coupled and therefore not discrete. Discrete eigenmodes were indeed observed [8], and the loading from these varied in time as plasma density changed during the discharge as predicted by the fast wave eigenmode analysis.

Clear ion heating was observed for short $(1.5 \mathrm{~ms}) \mathrm{RF}$ pulses at $\Omega_{\mathrm{d}} \approx 2$ with charge exchange systems: directed radially near the antenna and tangentially on the other side of the torus from the antenna (Fig. 4) [8]. The perpendicular energy distribution was bi-Maxwellian (Fig. 5) whereas the tangential distribution was a single Maxwellian. It was not clear at the time what caused the bi-Maxwellian distribution since the charge exchange analyzer did not have mass discrimination and the minority heating effect was not yet known.

For longer RF pulses $(5.5 \mathrm{~ms})$ the parallel temperature time response was rather slow during the fall off after the pulse due to the effect of impurities induced by the RF energy [8]. This effect was reproduced approximately with a neon gas pulse set to produce a $\mathrm{Z}$ heating mode with the same density 


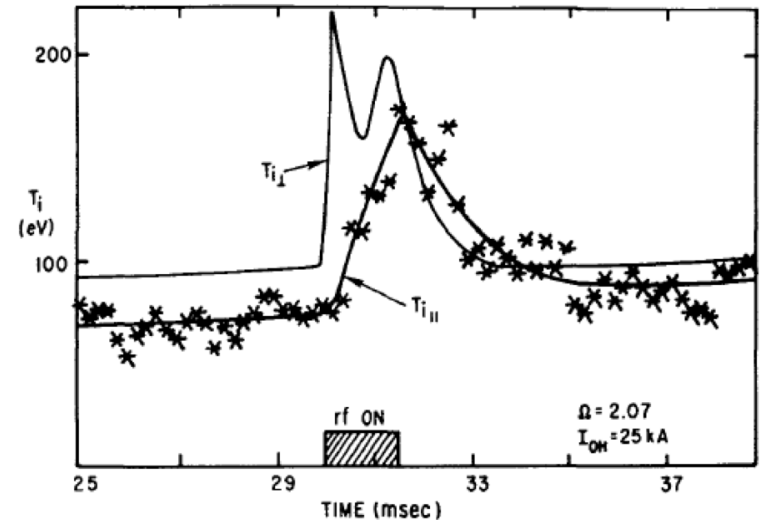

Figure 4. $\mathrm{T}_{\mathrm{ill}}$ and $\mathrm{T}_{\mathrm{i} \perp}$ versus time from charge exchange measurements in $\mathrm{D}_{2} .\left(\mathrm{P}_{\mathrm{RF}} \sim 70 \mathrm{~kW}, \mathrm{n}_{\mathrm{e}}=6 \times 10^{12} \mathrm{~cm}^{-3}, \Omega=\right.$ 2.07). (Fig. 10 of Ref. 8 with IAEA permission)

perturbation as the RF pulse [8]. An attempt was made to reduce the RF produced density by sealing off a Thomson scattering window leak and enclosing it in helium thinking that water vapor might be contributing to the observed impurity effect on density. In hindsight, the energetic minority ions were very poorly confined in ST, and even more so as the hydrogen

concentration was reduced with the water vapor leak at the Thomson scattering window displaced with helium. It was noticed that the RF

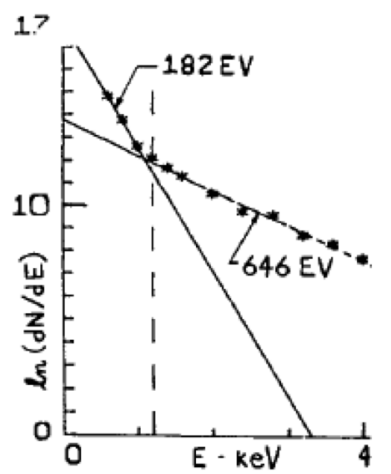

Figure 5. Perpendicular energy distribution induced at $\Omega=2$. (Fig. 9 of Ref. 8 with IAEA permission) substantially immediately following a vessel opening, presumably due to a greater hydrogen concentration resulting in a lower energy tail on the hydrogen energy distribution.

\section{PLT tokamak: Clear demonstration of the minority $\mathrm{H}$ regime, minority ${ }^{3} \mathrm{He}$ regime established, and second harmonic regime shown to be viable in hydrogen}

Prior to the start of ICRF experiments on PLT an important technology advance was made with the installation of a mass sensitive charge exchange system being developed for TFTR. The initial ICRF experiment was conducted with a modest RF power $(\sim 40 \mathrm{~kW})$ and the deuterium channel showed a relatively small temperature rise. An RF team member suggested looking also at the hydrogen channel, since we could, and it was discovered that the hydrogen energy distribution was greatly enhanced by the ICRF power and exhibited a very energetic tail. It was suddenly clear that the confusing heating results obtained on the ST tokamak were due to operating in the "minority" hydrogen regime. On PLT the energetic hydrogen confinement was no longer affected by severe bananaorbit loss cones as on the ST tokamak and the measured hydrogen energy extended to $\sim 40 \mathrm{keV}$ at $\mathrm{P}_{\mathrm{RF}}$ of only $~$ $40 \mathrm{~kW}$ (Fig. 6) [9]. The theoretical curve is for $\mathrm{Z}_{\text {eff }}=$ 2.2, $\mathrm{E}_{\mathrm{j}}=1.8 \mathrm{keV}$, and $\xi=13.8$ [9]. As $\mathrm{P}_{\mathrm{RF}}$ was subsequently increased to $\sim 350 \mathrm{~kW}, \mathrm{~T}_{\mathrm{d}}$ was increased substantially to $\sim 1.21 \mathrm{keV}$ from the ohmic level of $\sim$ $0.54 \mathrm{keV}$ [10], thereby establishing that the minority $\mathrm{H}$ regime was an effective heating method when the energetic $\mathrm{H}$ ions were adequately confined.

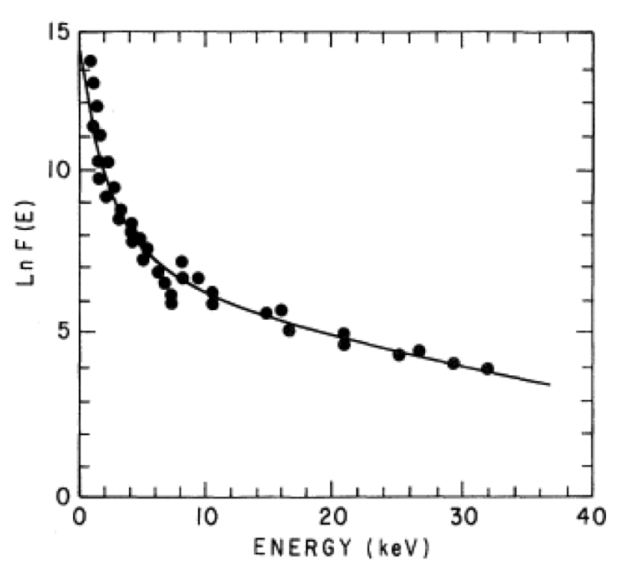

Figure 6. Hydrogen charge exchange spectra $20 \mathrm{~ms}$ into a $\sim 40 \mathrm{~kW}$ RF pulse for initial ICRF operation at $25 \mathrm{MHz}$ on PLT. (Fig. 4 of Ref. 9)

A technological event occurred - an internal turn-toturn short in a toroidal field coil - and PLT machine operations were stopped for several months. We used this time to explore possible minority species that could be used to optimize minority heating near the maximum $\mathrm{B}_{\mathrm{T}}$ available after the coil repair $-\sim 3 \mathrm{~T}$. This search resulted in the selection of helium-3 $\left({ }^{3} \mathrm{He}\right)$ as the minority species for which our $30 \mathrm{MHz}$ system frequency matched the cyclotron frequency at $3 \mathrm{~T}$. This ${ }^{3} \mathrm{He}$ minority regime was analyzed to be $\sim 65 \%$ more efficient at heating deuterium ions over the electrons than for the $\mathrm{H}$ minority regime [10]. This was born out experimentally by comparing the scaling of $\Delta \mathrm{T}_{\mathrm{d}}$ with $\mathrm{P}_{\mathrm{RF}} / \mathrm{n}_{\mathrm{e}}$ for the two minority regimes [10].

In order to push to higher ICRF power on PLT, it was critical to develop a high power alumina cone feedthrough which eliminated multipacting and allowed

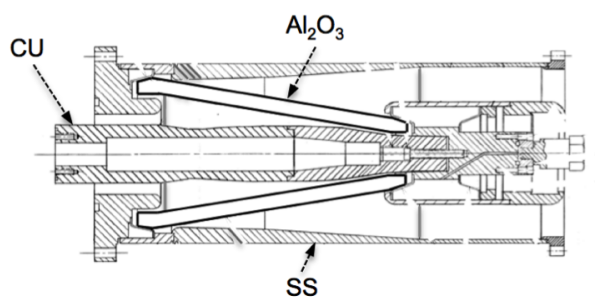

Figure 7. Alumina cone feedthrough developed to support high power operation on PLT. 
high voltage operation without multi-pacting (Fig. 7) [11]. With this feedthrough installed, the RF power was increased to $4.3 \mathrm{MW}$ and very large increases in $\mathrm{T}_{\mathrm{d}}$ and $\mathrm{T}_{\mathrm{e}}$ were produced in the $\mathrm{D} /{ }^{3} \mathrm{He}$ minority regime (Fig. 8) [12].

The second harmonic heating regime was also shown to be viable on PLT. Second harmonic heating of a hydrogen plasma with $\mathrm{P}_{\mathrm{RF}}$ at the $1 \mathrm{MW}$ level was

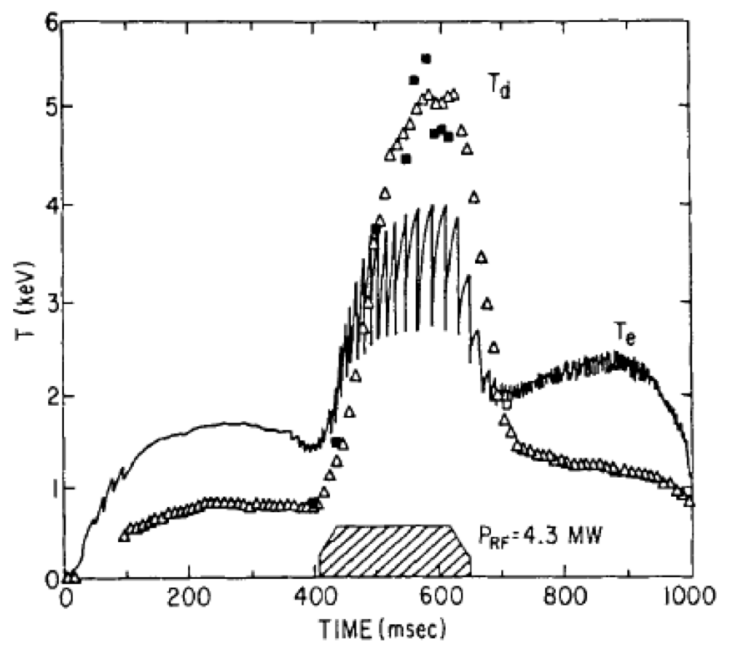

Figure 8. Time evolution of $T_{e}(0)$ (solid line) and bulk $T_{d}$ (charge exchange $\Delta$ and neutron emission $\square$ ) for a $\mathrm{D} /{ }^{3} \mathrm{He}$ case $\left[\mathrm{P}_{\mathrm{RF}}=4.3 \mathrm{MW}\right.$ at $30 \mathrm{MHz}, \mathrm{B}_{\mathrm{T}}=3.3 \mathrm{~T}, \mathrm{n}_{\mathrm{e}}=3.7 \times 10^{13} \mathrm{~cm}^{-3}$, $\eta_{3 \mathrm{He}}=5-10 \%$ ]. (Fig.1 of Ref. 12 with AIP Publishing permission)

(a)

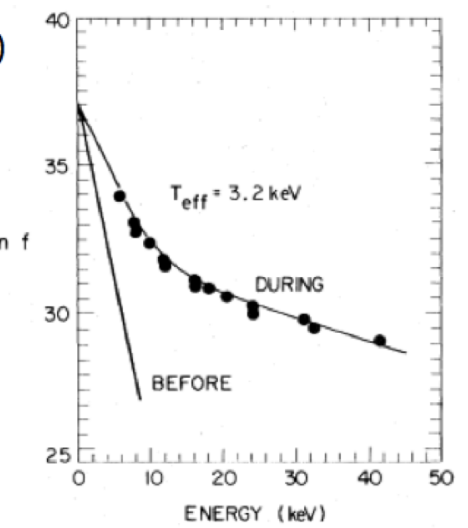

(b)

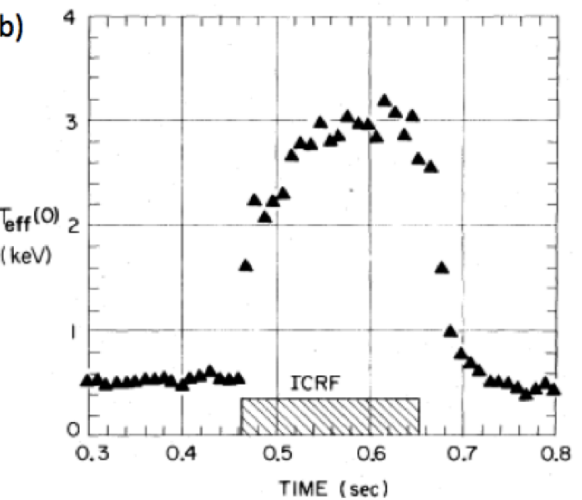

Figure 9. a) Hydrogen ion energy distribution before and during ICRF heating $\left(\mathrm{P}_{\mathrm{RF}} \sim 1 \mathrm{MW}, \mathrm{n}_{\mathrm{e}}=3.8 \times 10^{13} \mathrm{~cm}^{-3}\right)$, and $b)$ time evolution of average energy, $\mathrm{T}_{\text {eff }}=2 / 3\langle E\rangle$. (Fig. 1 of Ref. 13) successful in increasing the hydrogen effective temperature $\left[T_{\text {eff }}=2 / 3\langle E\rangle\right]$ from $\sim 0.5 \mathrm{keV}$ to $\sim 3 \mathrm{keV}$ (Fig. 9) [13]. This is an important method that can be used in the low-B startup phase of ITER and for the second harmonic $\mathrm{T}$ regime in a D-T plasma.

\section{TFTR tokamak: Second harmonic tritium regime demonstrated, and efficient mode conversion heating and current drive established}

ICRF experiments were carried out on TFTR in the minority hydrogen regime prior to the introduction of tritium for $\mathrm{P}_{\mathrm{RF}}$ up to $8.4 \mathrm{MW}$ [14]. Electron heating resulted in a large increase in $\mathrm{T}_{\mathrm{e}}(0)$ from $\sim 2.4 \mathrm{keV}$ to 6 $\mathrm{keV}$. Also, above $3 \mathrm{MW}$ the sawteeth were stabilized or significantly lengthened during the RF pulse. The energetic $\mathrm{H}$ distribution was measured out to $1 \mathrm{MeV}$ energy and found to have a high effective temperature. A large increase in TAE mode amplitude was observed versus $\mathrm{P}_{\mathrm{RF}}$ and this later led to a vacuum failure during the D-T phase of operation due to the loss of energetic ions via banana trapping which bombarded exposed welds at the bottom ports on TFTR.

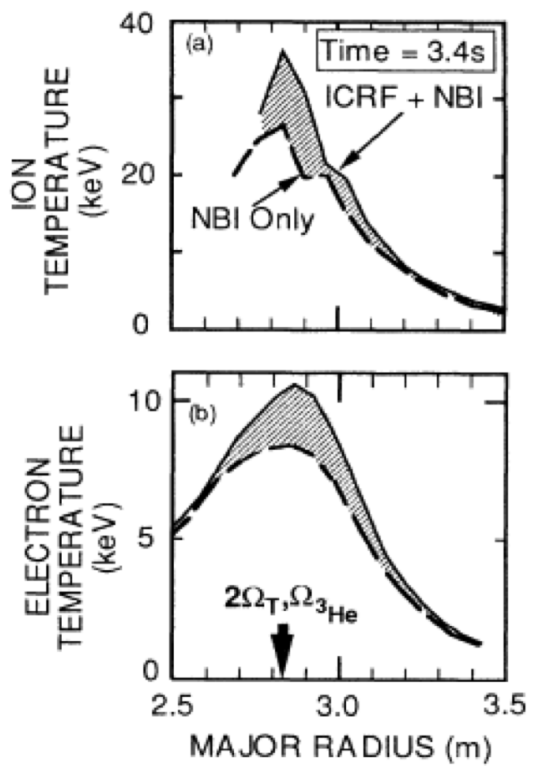

Figure 10. Ion and electron temperature profiles showing strong ICRF central ion and electron heating at $2 \Omega_{\mathrm{CT}}$ on TFTR. (Fig. 6 of Ref. 15)

In the first D-T ICRF experiments on a tokamak, substantial ion and electron temperature increments were obtained with $2 \Omega_{\mathrm{ct}}$ damping in hot ion mode discharges as shown in Fig. $10[15,16]$. For this case of $\mathrm{P}_{\mathrm{RF}}=5.5$ $\mathrm{MW}$ with $\mathrm{P}_{\mathrm{NBI}}=23.5 \mathrm{MW}(60 \% \mathrm{~T})$, the ICRF majority tritium heating at $2 \Omega_{\mathrm{ct}}$ resulted in temperature increments of $\Delta \mathrm{T}_{\mathrm{i}}(0)=9 \mathrm{keV}$ and $\Delta \mathrm{T}_{\mathrm{e}}(0)=2.6 \mathrm{keV}$. It is to be noted that a small amount of ${ }^{3} \mathrm{He}$ minority, which has a cyclotron frequency equal to $2 \Omega_{\mathrm{cT}}$, was also present in the discharge. The majority $2 \Omega_{\mathrm{cT}}$ /minority ${ }^{3} \mathrm{He}$ heating regime is ideal for heating in the reactor $\mathrm{D}-\mathrm{T}-{ }^{3} \mathrm{He}$ plasma 
since damping on the ${ }^{3} \mathrm{He}$ can serve to heat the tritium to a temperature level at which the $2 \Omega_{\mathrm{cT}}$ heating will take over even in the absence of neutral beam ion heating.

Mode conversion heating and current drive with outside launch were also demonstrated on TFTR $[17,16]$. The electron heating for a ${ }^{3} \mathrm{He}-{ }^{4} \mathrm{He}$ case with $\mathrm{P}_{\mathrm{RF}}=4.4$ MW resulted in $T_{e}(0)$ doubling to $6 \mathrm{keV}$ and $T_{e}$ approximately doubling across the profile as well. The measured electron power deposition profile peaks off axis at the mode conversion layer as predicted.

\section{NSTX tokamak: Transition to high harmonic fast wave (HHFW) regime and close examination of SOL losses}

The AORSA and GENRAY codes for HHFW launched at the plasma separatrix predicted very strong RF power absorption in the plasma core on NSTX with the wave power damping being $\sim 80 \%$ in approximately $1 / 4$ turn around the torus [18]. This strong "single pass" absorption serves to accentuate the competition between core RF power deposition and RF power loss in the SOL. It was immediately observed that the HHFW heating efficiency was optimum when the density in front of the antenna in the SOL was below the fast wave onset density for the $\mathrm{k}$ value launched by the antenna (set by phase between antenna straps) [18]. The heating efficiency $\left(\mathrm{W}_{\mathrm{T}}\right.$ at constant $\left.\mathrm{P}_{\mathrm{RF}}\right)$ falls off for phasing below $90^{\circ}$ for the conditions studied [18]. This result suggests strong RF power loss occurs in the SOL for $\mathrm{k}$ values that put the density in front of the antenna above the fast wave cut-off density, $\mathrm{n}_{\mathrm{co}} \propto \mathrm{B}^{*} \mathrm{k}^{2} / \omega$.

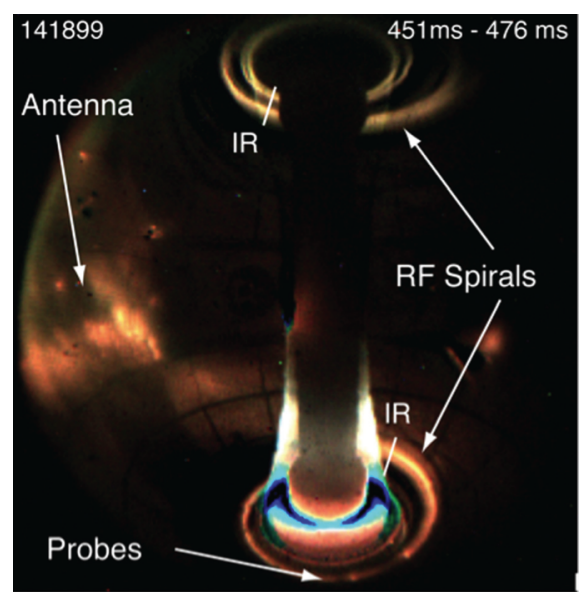

Figure 11. RF spirals produced on the bottom and top divertor plates of NSTX with HHFW heating $\left(\mathrm{P}_{\mathrm{RF}}=1.3\right.$ $\left.\mathrm{MW}, \mathrm{P}_{\mathrm{NB}}=2 \mathrm{MW}, \mathrm{D}\right)$. (Fig. 1 of Ref. 21 with AIP Publishing permission)

HHFW heating in L-mode plasmas was strong when the edge density was low relative to the cut off density, producing $\mathrm{T}_{\mathrm{e}}(0)$ values of $\sim 5 \mathrm{keV}$ in both $\mathrm{He}$ and $\mathrm{D}$ plasmas [19]. HHFW heating in ELM free H-mode plasmas showed significant heating toward the periphery of the plasma core for antenna strap phasings of $-150^{\circ}$ $\left(\mathrm{k}_{\phi} \sim 14 \mathrm{~m}^{-1}\right)$ and $-90^{\circ}\left(\sim-8 \mathrm{~m}^{-1}\right)$ [20]. Here the core heating efficiencies $\left(\eta=\mathrm{P}_{\text {dep }} / \mathrm{P}_{\text {applied }}\right.$, with $\mathrm{P}_{\text {dep }}$ measured from $\Delta \mathrm{W}_{\mathrm{T}} / \tau$ measurements) were $66 \%$ and $40 \%$ for $-150^{\circ}$ and $-90^{\circ}$, respectively. This indicates a large RF power loss in the SOL that increases inversely with $\mathrm{k}_{\triangleleft}$, as might be expected since the cut off density decreases as $\mathrm{k}_{\varphi}^{2}$.

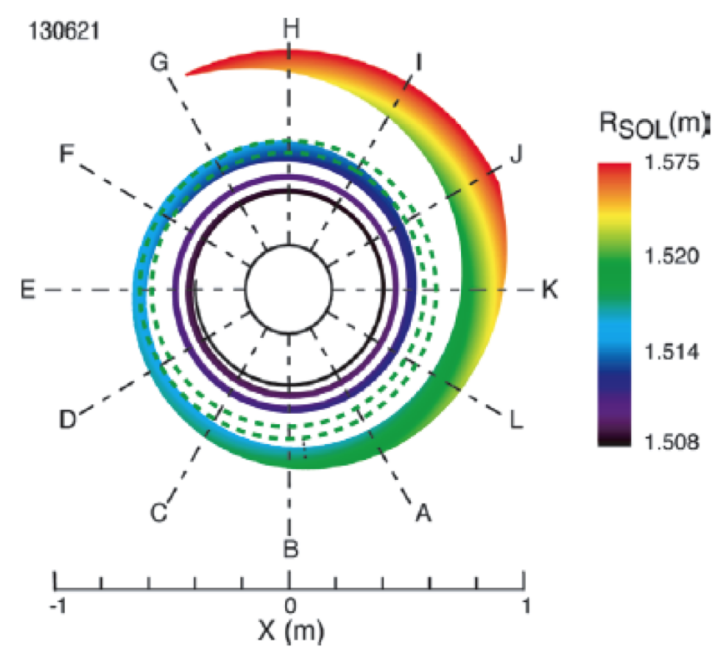

Figure 12. Magnetic field strike points on the lower divertor versus midplane field line $\mathrm{R}_{\mathrm{SOL}}$ location in front of the antenna. (Fig. 4 of Ref. 23 with IAEA permission)

It came as a surprise to us that much of the power loss to the SOL ended up on the top and bottom divertors in the form of "hot" spirals as shown in Fig. 11 [21]. This edge loss is reminiscent of the power loss to the SOL on the $\mathrm{C}$ stellarator. Considerable effort has been applied to understanding these spiral patterns. The SPIRAL code was used to map the magnetic field lines passing in the SOL in front of the antenna at the midplane to their strike points on the divertors, and indeed these strikepoints form a spiral (Fig. 12) $[22,23]$. The passes of this spiral line up with the IR camera peaks observed along a major radius view of the divertor floor [23]. Furthermore, the RF effects for the lower spiral are observed on probe floating potentials and instrumented tile currents [22]. The negative shift of the probe floating potential for the probe under the spiral is consistent with RF rectification for the far RF fields

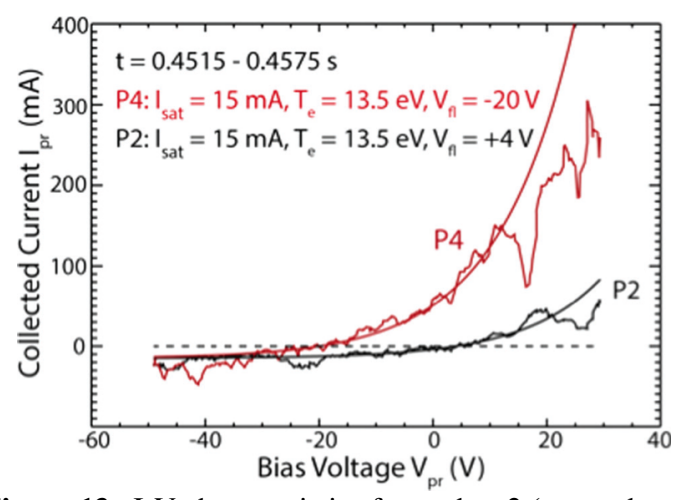

Figure 13. I-V characteristics for probes 2 (not under spiral) and 4 (under the spiral) with $\mathrm{P}_{\mathrm{RF}}=1.3 \mathrm{MW}$, averaged over $6 \mathrm{~V}_{\mathrm{pr}}$ sweeps. (Fig. 6 of Ref. 21 with AIP Publishing permission) 
arriving at the divertor along the magnetic field lines [21]. Also, for the tiles under the spiral the RF induced currents are caused by electron collection which is also consistent with RF rectification. Upon averaging the probe characteristics over six consecutive voltage sweeps, the negative shift of the I-V characteristic under the spiral is as expected for RF rectification as well (Fig. 13) [21]. For Fig. 13, the RF voltage across the probe sheath consistent with the $\mathrm{V}_{\mathrm{fl}}$ shift is $\mathrm{V}_{\mathrm{RF}}=43.7 \mathrm{~V}$.

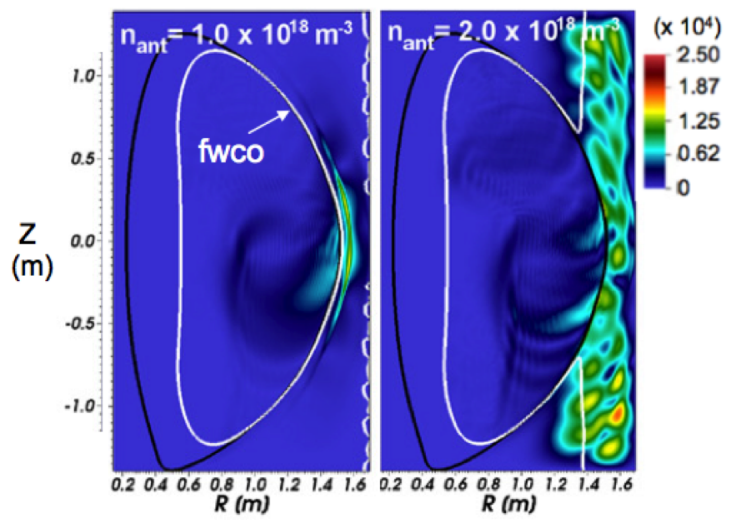

Figure 14. AORSA prediction of RF electric field increase in the SOL versus density at the antenna for $\mathrm{n}_{\odot}=-21$. The enhanced SOL field occurs when the FW cutoff (fwco) begins to open in front of antenna. (Fig. 2 of Ref. 24 with IAEA permission)

Finally, the AORSA code has been extended to include waves in the SOL and compared to the results obtained on NSTX [24]. Strong standing wave RF electric field amplitudes in the SOL are predicted for edge densities in front of the antenna above the fast wave cutoff density. This is illustrated clearly in Fig. 14 where the strong fields are predicted when the density at the antenna is raised above the FW cutoff density in front of the antenna causing the fast wave cutoff to open in front of the antenna. These enhanced fields are thought to be responsible for the observed far field rectification at the divertor.

\section{Summary}

The evolution of understanding of ICRF regimes on the C stellarator through to the NSTX tokamak with experiments, modeling and technology advances has been summarized. Much is now understood from this research and research on other devices in the world fusion program. It is generally recognized that far field wave fields away from the antenna and near field wave fields in the vicinity of the antenna can cause considerable RF power loss and result in unwanted impurity production as well. Extensive efforts have been and are underway to quantify the near field and far field effects, and efforts are also underway to modify antenna designs to reduce these fields. Particularly noteworthy recent antenna advances include the field aligned antenna on C-mod [25] and the 3-strap antenna on ASDEX Upgrade [26].
The authors wish to acknowledge the support of the several fusion machine project teams, and especially the machine, RF, and neutral beam operations groups. This work is supported by USDOE Contract No. DE-AC02-09CH11466.

\section{References}

1. M.A. Rothman, R.M. Sinclair, I.G. Brown, and J.C. Hosea, Phys. Fluids 12, 2211 (1969)

2. T.H. Stix, Theory of Plasma Waves (McGraw-Hill Book Company, New York, 1962)

3. M.A. Rothman, R.M. Sinclair, and S. Yoshikawa, J. Nucl. Ener. Part C_Plasma Physics 8, 241 (1966)

4. J.C. Hosea and R.M. Sinclair, PRL 23, 3 (1969)

5. J.C. Hosea and R.M. Sinclair, Phys Fluids 13, 701 (1970)

6. J.C. Hosea and R.M. Sinclair, Phys. Fluids 16, 1268 (1973)

7. J.C. Hosea and W.M. Hooke, PRL 31, 150 (1973)

8. J. Adam, M. Chance, H. Eubank, et al., $5^{\text {th }}$ IAEA Fusion Energy Conf Vol 1, 65 (Tokyo, November 1974)

9. J. Hosea, S. Bernabei, P. Colestock, et al., PRL 43, 1802 (1979)

10. J. Hosea, V. Arunasalam, S. Bernabei, et al., Proc. Course on Physics of Plasmas Close to Thermonuclear Conditions Vol 2, 571 (Varenna, August-September 1979)

11. D.Q. Hwang, G. Grotz, and J.C. Hosea, J. Vac. Sci. and Technol. 28, 1273 (1982)

12. J.R. Wilson and the PLT Group, RF Conf. Proc. AIP 129, 28 (1985)

13. D.Q. Hwang, J. Hosea, H. Thompson, et al., PRL 51, 1865 (1983)

14. C.K. Phillips, J.R. Wilson, M. Bell, et al., RF Conf. Proc. AIP 289, 44 (1994)

15. J.R. Wilson, C.E. Bush, D. Darrow, et al., PRL 75, 842 (1995)

16. C.K. Phillips, M.G. Bell, R. Bell, et al., Phys. Plasmas 2, 2427 (1995)

17. R. Majeski, J.H. Rodgers, S.H. Batha, et al., PRL 76, 764 (1996)

18. J. Hosea, R.E. Bell, B.P. LeBlanc, et al., Phys. Plasmas 15, 056104 (2008)

19. G. Taylor et al., RF Conf. Proc. AIP 1187, 113 (2009)

20. J.C. Hosea, R.E. Bell, E. Feibush, et al., RF Conf. Proc. AIP 1187, 105 (2009)

21. R.J. Perkins, J.C. Hosea, M.A. Jaworski, et al., Phys. Plasmas 22, 042506 (2015)

22. R.J. Perkins, J.C. Hosea, G.J. Kramer, et al., PRL 109, 045001 (2012)

23. R.J. Perkins, J.-W. Ahn, R.E. Bell, et al., Nucl. Fusion 53, 083025 (2013)

24. N. Bertelli, E.F. Jaeger, J.C. Hosea, et al., Nucl. Fusion 54, 083004 (2014)

25. S.J. Wukitch, M.L. Garrett, R. Ochoukov, et al., Phys. Plasmas 20, 056117 (2013)

26. V. Bobkov, F. Braun, R. Dux, et al., Nucl. Fusion 56, 084001 (2016) 